\title{
Abordagens faunísticas e geoquímicas em microfósseis calcários e suas aplicações à paleoceanografia e paleoclimatologia
}

\author{
The faunal and geochemical approaches in the calcareous microfossils and their \\ applications to the palaeoceanography and palaeoclimatology
}

\author{
Cristianini Trescastro Bergue \\ joão Carlos Coimbrall
}

Resumo: A micropaleontologia é uma importante aliada à pesquisa paleoceanográfica e paleoclimática, contribuindo para o estudo de parâmetros, como taxas de sedimentação, variações do nível relativo dos oceanos e mudanças hidrológicas (circulação, temperatura, produtividade e salinidade, por exemplo). Este artigo de revisão objetiva apresentar algumas potencialidades e limitações de três importantes grupos de microfósseis calcários: nanofósseis, foraminíferos e ostracodes. Abordagens envolvendo a composição de espécies e geoquímica nestes grupos, no Cretáceo e Cenozóico, são aqui discutidas com base em estudos recentes e, sempre que possível, com exemplos de pesquisas desenvolvidas no Brasil.

Palavras-chave: Paleoceanografia. Paleoclimatologia. Microfósseis. Nanofósseis calcários. Foraminíferos. Ostracodes.

Abstract: Micropalaeontology is an important ally to the palaeoceanography and palaeoclimatology contributing to the study of factors such as sedimentation rates, sea level and hydrological changes (e.g. circulation, temperature, productivity and salinity). This revision article aims to present the main applications of three important groups of calcareous microfossils: nannofossils, foraminifers and ostracodes. Species composition and geochemical approaches on those groups both in Cretaceous and Cenozoic are discussed based on studies published in the last years, referring whenever it possible to examples of studies developed in Brazil.

Keywords: Palaeoceanography. Palaeoclimatology. Microfossils. Calcareous nanofossils. Foraminifers. Ostracodes.

Universidade do Vale do Rio dos Sinos. Laboratório de Micropaleontologia. São Leopoldo, Rio Grande do Sul, Brasil (cbergue@ unisinos.br).

" Universidade Federal do Rio Grande do Sul. Departamento de Paleontologia e Estratigrafia. Porto Alegre, Rio Grande do Sul, Brasil (joao.coimbra@ufrgs.br).

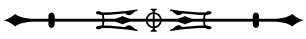




\section{INTRODUÇÃO}

Os microfósseis calcários marinhos compõem uma categoria de elementos originados de diferentes grupos animais e protistas. Embora menos conhecidos popularmente que os macrofósseis, constituem excelente fonte de dados para abordagens paleontológicas aplicadas, dentre as quais a paleoceanografia e paleoclimatologia.

Há um conjunto de características que os torna importantes neste tipo de pesquisa, ora compartilhadas, ora exclusivas de alguns grupos. Dentre elas, destacam-se:

a) a notável abundância, em alguns casos, excedendo milhares de indivíduos em poucos gramas de sedimento;

b) a composição carbonática, que viabiliza a realização de análises geoquímicas, especialmente elementos-traço e isótopos estáveis;

c) grande sensibilidade aos parâmetros ambientais, expressa por meio da variabilidade intraespećfica ou de mudanças na composição de espécies;

d) existência de táxons com longa distribuição temporal que permitem o estabelecimento de correlações atualísticas.

\section{MudANÇAS CLIMÁTICAS}

O oceano e a atmosfera formam um sistema integrado com papel determinante no clima da Terra, devido à atuação de ambos no transporte de calor. Portanto, é difícil estudar de forma dissociada eventos paleoceanográficos sem considerar o contexto paleoclimático, e vice-versa. Considerando que nosso planeta foi modelado ao longo do tempo pela interação entre seus componentes abióticos e bióticos, as mudanças climáticas em nível global estiveram sempre presentes na regulação deste sistema (Lovelock, 2006). É possível constatar isto por meio do registro geológico, o qual evidencia processos como o avanço e recuo de geleiras, variações de temperatura, regressões e transgressões marinhas e mudanças na composição e circulação de massas d'água.
Dados recentes, porém, indicam que a atividade humana induziu mudanças no clima da Terra num ritmo mais acelerado, mensurável em escala histórica. Acredita-se que a continuidade deste processo possa comprometer a sobrevivência de muitas espécies, bem como diversos setores da atividade humana, o que desencadeou discussões a respeito de estratégias para reverter este panorama. Existe uma forte relação entre este tema e a micropaleontologia, pois muitas modelagens paleoclimáticas são baseadas em microfósseis. Além disso, o estudo de associações atuais de grupos com grande representatividade fóssil serve de subsídio para estas pesquisas, pois constituem parâmetros para efeitos da ação antrópica. Os microfósseis calcários são especialmente importantes na pesquisa paleoceanográfica em seções do Cretáceo e Cenozóico. Suas potencialidades, porém, tornam-se mais limitadas à medida que se recua no tempo, pois a diagênese influencia em graus variáveis o emprego de análises geoquímicas e a correta identificação dos táxons.

Este artigo de revisão tem por objetivo apresentar alguns conceitos básicos e potencialidades dos microfósseis calcários na pesquisa paleoceanográfica e paleoclimatológica. A vastidão do tema, contudo, exige uma abordagem restrita, motivo pelo qual são discutidos apenas tópicos mais relevantes sobre os nanofósseis calcários, foraminíferos e ostracodes. Entretanto, a maior parte das metodologias aqui referidas é igualmente aplicável a outros grupos, como os micromoluscos, por exemplo. Na medida do possível, são discutidos estudos realizados no Brasil, visando divulgá-los e incentivar novos pesquisadores nesta linha de pesquisa em nosso país.

\section{NANOFÓSSEIS CALCÁRIOS}

O termo 'nanofósseis calcários' inclui organismos de

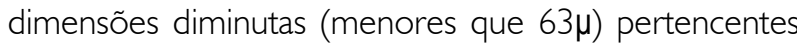
a diferentes grupos biológicos, principalmente cocolitoforídeos (protistas planctônicos). Também são rotineiramente incluídos neste grupo dinoflagelados calcários, espículas de ascídias e alguns organismos incertae

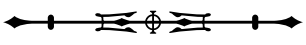


sedis, provavelmente de origem algálica. No registro fóssil, são mais abundantes os cocólitos (Figura 1A), pequenas placas do envoltório externo dos cocolitoforídeos. Os nanofósseis calcários originaram-se no Neotriássico e a primeira grande diversificação ocorreu no Jurássico. Desde então, são os principais fixadores de carbono atmosférico e importantes formadores de rochas carbonáticas.

Os níveis de abundância destes fósseis são excelentes indicadores de produtividade primária, havendo, ainda, outras potencialidades para a paleoceanografia e paleoclimatologia, devido ao papel que desempenharam na regulação climática durante o Cenozóico. Recentemente, constatou-se que reações metabólicas destes protistas produzem o sulfeto de dimetila, que atua como núcleo condensador de nuvens e, portanto, na regulação térmica da Terra através de sua influência no albedo.

\section{FORAMINÍFEROS}

Os foraminíferos (Figura 1B) são protistas marinhos ou transicionais que secretam uma carapaça carbonática ou aglutinante cuja morfologia constitui a base para a classificação do grupo. As formas bentônicas surgiram no Cambriano, porém, espécies planctônicas só ocorrem a partir do Jurássico. Estas, além do grande potencial bioestratigráfico, têm, ainda, imensa aplicabilidade paleoceanográfica, pois são importantes indicadores batimétricos (razão planctônicos/bentônicos) e marcadores de massas d'água devido à sensibilidade de algumas espécies à temperatura. As espécies bentônicas, por sua vez, são indicadoras das condições hidrológicas de fundo. Além disso, é em testas de foraminíferos que se realiza a maior parte dos estudos geoquímicos dentre os microfósseis.

\section{OSTRACODES}

Os ostracodes (Figura 1C-H) têm apresentado, nos últimos anos, crescente aplicação em paleoceanografia. Além do vasto espectro de ocorrência e abundância nos ambientes marinhos, existem táxons viventes com grande distribuição temporal, permitindo correlações atualísticas confiáveis.
A temperatura é o fator mais influente na distribuição das espécies, conforme constatado pela influência da termoclina na separação entre táxons de águas quentes (termofílicos) e de águas frias (criofilicos).

O conhecimento mais aprofundado sobre os padrões de distribuição batimétrica dos táxons, adquirido nas últimas décadas, ampliou o potencial paleobatimétrico e paleoidrológico do grupo. Constatou-se, ainda, que variações de riqueza e abundância estão geralmente associadas a mudanças na formação e circulação das massas d'água e da produtividade primária. Mais recentemente, o uso de análises geoquímicas difundiu-se entre os ostracodologistas, permitindo a análise simultânea de diversos parâmetros ambientais.

\section{DESVENDANDO A HISTÓRIA DOS OCEANOS: FONTES GEOQUÍMICAS E PALEONTOLÓGICAS}

Os dados em pesquisas paleoceanográficas podem ser obtidos por meio do estudo faunístico e/ou nanoflorístico (morfológicos e taxonômicos), ou pela análise quantitativa de determinados elementos nas carapaças, especialmente a razão isotópica $\left(\delta^{13} \mathrm{C}, \delta{ }^{18} \mathrm{O}\right)$. O critério de escolha de qual será empregado depende tanto dos objetivos do trabalho quanto de determinadas características das amostras, como a idade e o estado de preservação dos microfósseis. Em muitos casos, análises geoquímicas são valiosas e trazem importantes contribuições. É importante observar, porém, que estudos faunísticos, integrando tafonomia, diversidade e abordagem taxonômica acurada, podem ser tão ou mais informativos que os dados geoquímicos. Considerando que os táxons, em seu processo evolutivo, respondem de forma adaptativa a mudanças ambientais, através de especiação ou extinção, as variações de riqueza de espécies no tempo constituem também um excelente indicador oceanográfico. A união das abordagens geoquímica e paleontológica clássica, contudo, é recomendável sempre que possível.

As análises geoquímicas consistem, basicamente, na quantificação de elementos incorporados ao carbonato durante a precipitação da carapaça. A quantidade de um 


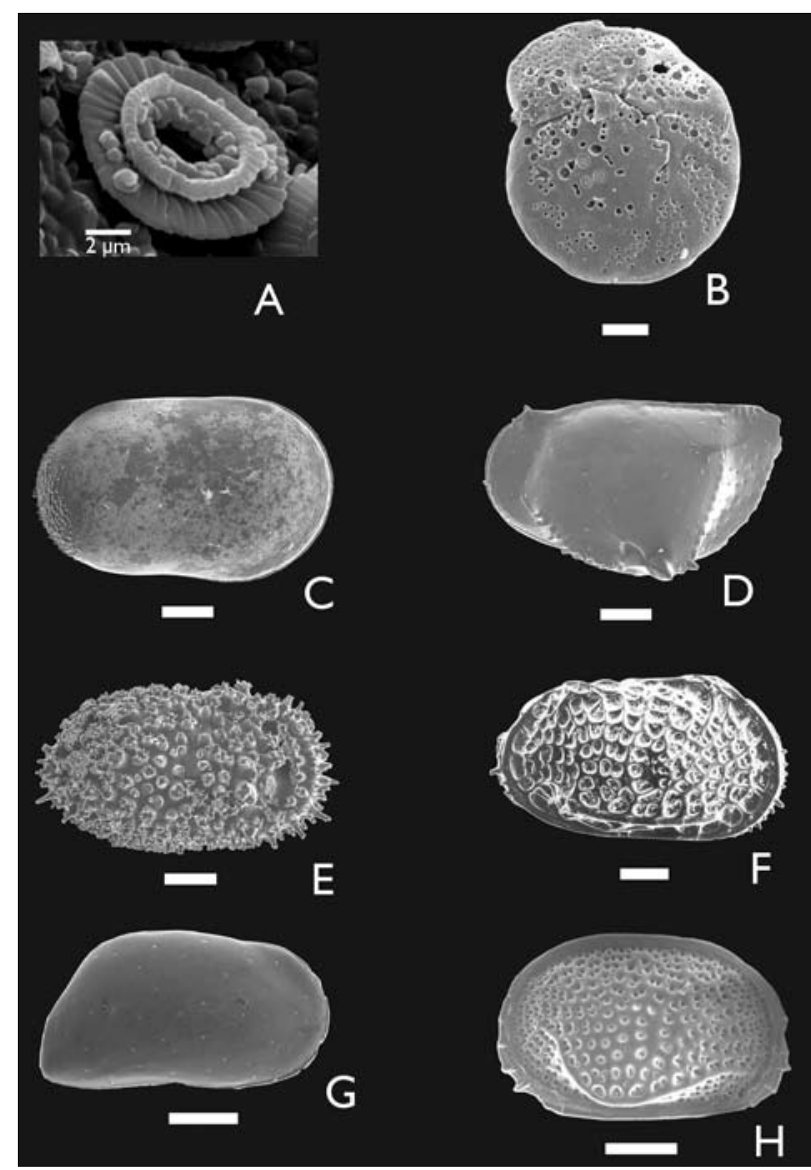

Figura 1. Alguns exemplos de microfósseis calcários. A: cocólito; B: Foraminífero. Cibicidoides sp.; C-H: Ostracodes. C: Cytherella serratula Brady; D: Bythocythere sp.; E: Trachyleberis sp.; F: Poseidonamicus pintoi Benson; G: Krithe dolichodeira Bold, 1946; H: Saida sp. (A, Mioceno ODP site 744A; B-H, Pleistoceno-Holoceno bacia de Santos).

determinado elemento, ou razão entre dois de seus isótopos estáveis, varia em função de determinados parâmetros ambientais, em especial a temperatura e a produtividade primária. Destes, a temperatura é o mais estudado, pela sua importância na dinâmica da circulação oceânica e atmosférica, explicando a ampla difusão dos chamados paleotermômetros, como o $\delta{ }^{18} \mathrm{O}$ e a razão $\mathrm{Mg} / \mathrm{Ca}$.

Um outro tipo de análise utilizada em paleotermometria, contudo, merece ser aqui discutido devido à sua relação com os nanofósseis calcários. A principal aplicação paleoceanográfica dos cocólitos é a caracterização térmica das massas d'água. Isto pode ser realizado através do estudo dos microfósseis propriamente ditos ou de moléculas orgânicas por eles produzidas, chamadas alquenonas, com cadeias de 37, 38 ou 39 carbonos. A produção de moléculas com insaturações duplas ou triplas é dependente da temperatura, sendo a redução da temperatura acompanhada pelo aumento da produção de alquenonas insaturadas. Estas moléculas ficam preservadas nos sedimentos e, através de sua medição, é possível inferir sobre a temperatura das águas superficiais.

\section{ANÁLISES GEOQUímICAS EM MICROFÓSSEIS CALCÁRIOS}

\section{ELEMENTOS-TRAÇO}

A potencialidade paleoceanográfica das análises de elementos-traço consiste não apenas em fornecer indícios sobre características físicas e químicas da água, mas também em proporcionar informações indiretas sobre variações do nível relativo do mar e circulação oceânica. As análises mais utilizadas em microfósseis calcários são as de estrôncio, magnésio e cádmio, indicadoras de salinidade, temperatura e produtividade, respectivamente. São muito utilizadas em foraminíferos, e a razão $\mathrm{Mg} / \mathrm{Ca}$, em especial, tornou-se recentemente bastante difundida em ostracodes.

O uso deste método como paleotermômetro fundamenta-se na gradual substituição de Ca por Mg na composição do carbonato, influenciada positivamente pela temperatura, conforme a relação $(\mathrm{Mg} / \mathrm{Ca})_{\text {calctata }}=$ $\mathrm{D}(\mathrm{Mg} / \mathrm{Ca})_{\text {agua }}$, sendo $\mathrm{D}$ o coeficiente de distribuição (Dwyer et al., 1995). Devido ao grande tempo de residência do magnésio nos oceanos, assume-se que a incorporação deste elemento no carbonato é controlada apenas pelo D. Estudos prévios demonstraram ainda que este coeficiente é influenciado pela ontogenia e peculiaridades metabólicas das diferentes espécies, exigindo ajustes metodológicos para sua utilização em cada gênero. Realizada a espectrometria, as paleotemperaturas são obtidas através de uma fórmula cujo resultado fornece seu valor em graus Celsius.

Apesar de alguns pesquisadores utilizarem gêneros de ostracodes como Bythocypris Brady, 1880 (ver Corrège \& De Deckker, 1997) e Loxoconcha Sars, 1866 (ver Cronin

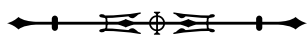


et al., 2005), Krithe Brady, Crosskey \& Robertson, 1874 ainda é o táxon tradicionalmente mais empregado. Isto é explicado pela grande abundância e distribuição de algumas espécies em águas profundas (>200 m). Esta característica ecológica favorece a comparação de dados oriundos de diferentes regiões. Dwyer et al. (1995) propuseram a fórmula $\mathrm{T}=0,854(\mathrm{Mg} / \mathrm{Ca})-$ 5,75 para o cálculo de paleotemperaturas em espécies deste gênero.

\section{IsÓTOPOS ESTÁVEIS}

Os isótopos estáveis, os mais utilizados na pesquisa micropaleontológica, recebem esta denominação porque a configuração dos seus núcleos não muda através do decaimento radioativo. Estas tradicionais análises vêm sendo utilizadas e aprimoradas nas últimas décadas e, embora outros isótopos sejam testados para este fim, os de carbono e oxigênio são os que fornecem melhores resultados.

Os isótopos de carbono são os indicadores de produtividade mais utilizados, fundamentando-se sua utilização numa peculiaridade metabólica dos organismos fotossintetizantes que incorporam preferencialmente $\mathrm{O}$ isótopo mais leve $\left({ }^{12} \mathrm{C}\right)$. Num contexto de alta produtividade oceânica, a água superficial fica enriquecida, portanto, com o isótopo mais pesado $\left({ }^{13} \mathrm{C}\right)$. Esta assinatura é registrada, por exemplo, nas carapaças dos organismos planctônicos. A matéria orgânica formada na superfície, por sua vez, ao se decompor no fundo oceânico, enriquece a água com o isótopo mais leve, sendo este sinal registrado nas carapaças da biota bentônica. Em síntese, pode-se dizer que, através dos sinais isotópicos em testas de foraminíferos planctônicos e bentônicos, avalia-se a eficiência da chamada 'bomba de carbono' oceânica.

Com relação ao oxigênio, o $\delta^{18} \mathrm{O}$ fornece informações diretas ou indiretas sobre temperatura, salinidade, volume de gelo e circulação oceânica. Sua utilização está baseada no estudo de Urey (1947), o qual demonstrou que os carbonatos apresentam diferentes razões entre ${ }^{18} \mathrm{O}$ e ${ }^{16} \mathrm{O}$, conforme a temperatura de precipitação. Contudo, sua aplicação micropaleontológica consagrou-se quando
Emiliani (1955) aplicou este princípio em foraminíferos planctônicos, corroborando o modelo de ciclicidade das variações orbitais proposto por outros autores. Os valores isotópicos plotados em um gráfico geraram uma curva cujos picos positivos e negativos (mais ou menos ${ }^{18} \mathrm{O}$, respectivamente) mostravam a ocorrência de períodos de clima quente e frio.

Naquele estudo, Emiliani (1955) subdividiu o Quaternário em estágios isotópicos marinhos (EIM), codificados em seqüência numérica crescente, onde os números ímpares correspondem aos períodos interglaciais e os pares aos glaciais. Exemplificando, o interglacial atual é representado pelo número um, o último máximo glacial (UMG) pelo número dois, e assim sucessivamente. Estudos posteriores produziram curvas isotópicas mais acuradas que permitiram, inclusive, a subdivisão dos EIM (Pisias et al., 1984).

A abundância natural do isótopo ${ }^{16} \mathrm{O}$ é de $99,76 \%$ e a do isótopo ${ }^{18} \mathrm{O}$ é de apenas $0,19 \%$, porém, estas proporções oscilam entre períodos glaciais e interglaciais. Durante os glaciais, a água do mar encontra-se enriquecida com o isótopo mais pesado (picos positivos). Ao mesmo tempo, a água depositada nas geleiras encontra-se enriquecida com o isótopo mais leve, $0{ }^{16} \mathrm{O}$. Isso ocorre porque o isótopo leve é extraído mais facilmente por meio da evaporação e migra para as altas latitudes, onde fica retido nas calotas polares, principalmente. Ao término dos períodos glaciais, o derretimento em larga escala destas massas de gelo restabelece a proporção natural entre os dois isótopos (picos negativos de ${ }^{18} \mathrm{O}$ ).

A razão molar de um isótopo num carbonato pode refletir ou não a existente na água (precipitação em equilíbrio ou desequilíbrio, respectivamente). A precipitação em desequilíbrio é decorrente do chamado efeito vital e possivelmente reflete peculiaridades metabólicas dos organismos, motivo pelo qual alguns grupos fósseis são melhores do que outros para este tipo de análise. A razão isotópica é medida em espectrometria através da dissolução das carapaças em ácido fosfórico (processo de 
extração) e subseqüente análise dos gases liberados. $\bigcirc$ valor obtido é comparado com um padrão (geralmente o PDB), o qual, na prática, é calibrado com o uso de um carbonato padrão sintético, o NBS-19 (Equação 1). A razão obtida serve de base para cálculos de paleotemperatura (Equação 2) ou paleosalinidade (Equação 3), sendo as equações ajustadas conforme a região estudada.

\section{Equação 1}

$\delta^{18} \mathrm{O}=\left(\mathrm{R}_{\text {amostra }}-\mathrm{R}_{\text {padräa }}\right) \cdot 10^{3} / \mathrm{R}_{\text {padrão }}$, sendo $\mathrm{R}={ }^{18} \mathrm{O} /{ }^{16} \mathrm{O}$

\section{Equação 2}

$\mathrm{T}=16,1-4,64\left(\delta^{18} \mathrm{O}_{\text {carbonato }}-\delta^{18} \mathrm{O}_{\text {água }}\right)+0,09\left(\delta^{18} \mathrm{O}_{\text {carbonato }}\right.$ $\left.-\delta{ }^{18} \mathrm{O}_{\text {água }}\right)$

\section{Equação 3}

$\mathrm{S}=2,334 . \delta^{18} \mathrm{O}+34,66$

As análises de isótopos estáveis são realizadas em foraminíferos planctônicos e bentônicos, dependendo se o objetivo é o estudo de massas d'água superficiais ou de fundo. Globigerina e Globigerinoides estão entre os foraminíferos planctônicos mais abundantes, constituindo uma importante fonte de carbonato para análises geoquímicas. Os bentônicos Cibicidoides e Uvigerina são também gêneros bastante conhecidos ecologicamente e amplamente empregados em estudos paleoambientais, sendo o último caracterizado por hábito infaunal. Nestes gêneros, o efeito vital é inexistente ou pouco pronunciado, o que os torna adequados para estudos geoquímicos. Recentemente, Costa et al. (2006) estabeleceram uma nova relação entre $\delta{ }^{18} \mathrm{O}$ e salinidade para o setor ocidental do Atlântico Sul, com base em adaptações de fórmulas propostas por outros autores para os gêneros Cibicidoides e Uvigerina. Comparando dados de salinidades atuais com os sinais isotópicos obtidos nestes gêneros, concluíram que o primeiro produz resultados mais próximos do equilíbrio (efeito vital menor) do que Uvigerina, sendo mais recomendável, portanto, para aplicações paleoceanográficas.
Estudos recentes têm difundido a análise de isótopos estáveis também em ostracodes quaternários. Didié \& Bauch (2002) analisaram os gêneros Henryhowella e Krithe, e Mazzini (2005) analisou isótopos de carbono e oxigênio, comparativamente, nos gêneros Krithe, Fallacihowella, Poseidonamicus e Bradleya. A importância destes trabalhos para a geoquímica dos ostracodes consiste em terem sido realizados em material quaternário cujos parâmetros ambientais são relativamente bem conhecidos. Seus resultados permitem, portanto, avaliar a influência de fatores ecológicos - como o hábito infaunal - na assinatura isotópica da carapaça e sua extrapolação para amostras mais antigas. Estes incipientes estudos apontam que o efeito vital existente nos ostracodes (offset positivo) é mais pronunciado que aquele dos foraminíferos, mas não invalida sua utilização como fonte adicional de dados (Figura 2).

Os elementos-traço e os isótopos estáveis podem ser analisados de forma concomitante no mesmo grupo fóssil ou em grupos distintos (Figuras 3 e 4). Autores como Lear et al. (2000) sugerem que a combinação de análises isotópicas e de elementos-traço fornece resultados mais consistentes, pois, diferentemente do $\delta^{18} \mathrm{O}$, a quantidade do íon magnésio nos oceanos não é alterada pela formação das massas de gelo. Sua precipitação na carapaça reflete, portanto, apenas influência da temperatura.

\section{Mudanças nas associações Fósseis}

Intervalos de abundância ou riqueza de ostracodes, foraminíferos e nanofósseis, bem como variações intraespecíficas, podem ser indicadores de mudanças hidrológicas. Estes organismos têm associações distintas em ambientes costeiros, neríticos e batiais, definidas basicamente pela salinidade e temperatura da água.

Os foraminíferos são os microfósseis bentônicos mais abundantes e conhecidos quanto ao seu aspecto ecológico. Existem táxons como Ammonia e Elphidium, típicos de águas rasas, enquanto Cibicidoides, Epistominella e Nutallides, por exemplo, são característicos de ambientes batiais. Através 


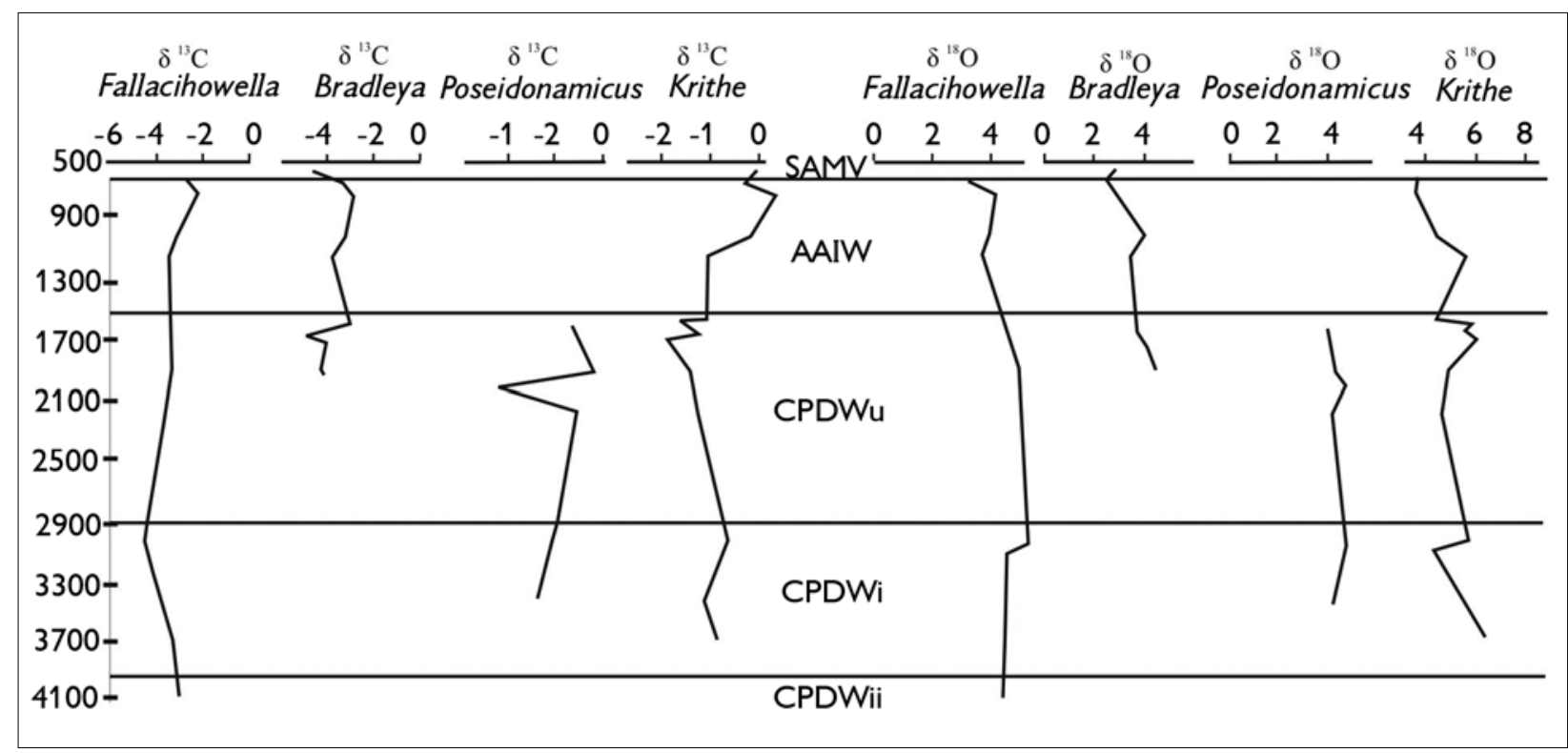

Figura 2. Curvas comparativas de isótopos de oxigênio e carbono em ostracodes marinhos do Quaternário. Modificado de Mazzini (2005).

do estudo de aspectos tafonômicos e taxonômicos, é possível realizar uma boa caracterização ambiental. Lançone et al. (2005) propuseram a caracterização hidrológica da enseada do Flamengo, no litoral de São Paulo, com base neste princípio. Na região batial, os fatores mais influentes na distribuição das faunas parecem variar conforme a região considerada. Alguns autores, como Thomas et al. (1990), demonstraram que, na porção ocidental do Atlântico Norte (Nova Scotia), as associações de espécies modificam-se conforme as massas d'água presentes. Na bacia de Campos, por outro lado, Sousa et al. (2006) demonstraram que o suprimento de matéria orgânica e a sedimentologia são os fatores mais importantes na definição das associações.

A caracterização ecológica dos táxons é um método seguro de inferência paleobatimétrica, contudo sua utilização deve ser feita com cautela, pois as carapaças estão sujeitas a processos de transporte. Carapaças depositadas próximo à quebra da plataforma, por exemplo, podem ser transportadas para o talude por ação de desmoronamentos ou suspensão por correntes de turbidez. Uma outra situação possível é o retrabalhamento de sedimentos da plataforma ao longo dos ciclos transgressivos/regressivos e sua deposição no talude. Ambos os casos acima exemplificados provocam a mistura de elementos de águas rasas e profundas, podendo gerar interpretações paleobatimétricas equivocadas.

Os picos de abundância são também valiosas fontes de dados e podem ser inferidos pelas taxas de acumulação (BFAR - 'Benthic Foraminiferal Accumulation Rates'). Estas taxas oscilam conforme mudanças na produtividade primária e taxas de sedimentação, podendo diferir, entretanto, entre táxons epifaunais e infaunais. Outro fator influente é o grau de preservação das testas, as quais estão sujeitas a dissolução em decorrência de modificações na profundidade da lisoclina.

Alguns foraminíferos planctônicos, como Globorotalia, são bons indicadores térmicos, pois o sentido do enrolamento das testas (dextrógiro ou levógiro) varia conforme a temperatura da água. O gênero de cocolitoforídeo Gephyrocapsa também apresenta variações morfológicas nos cocólitos em função da temperatura. Esta sensibilidade permite que espécies planctônicas sejam também utilizadas como marcadores

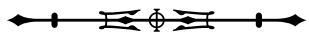




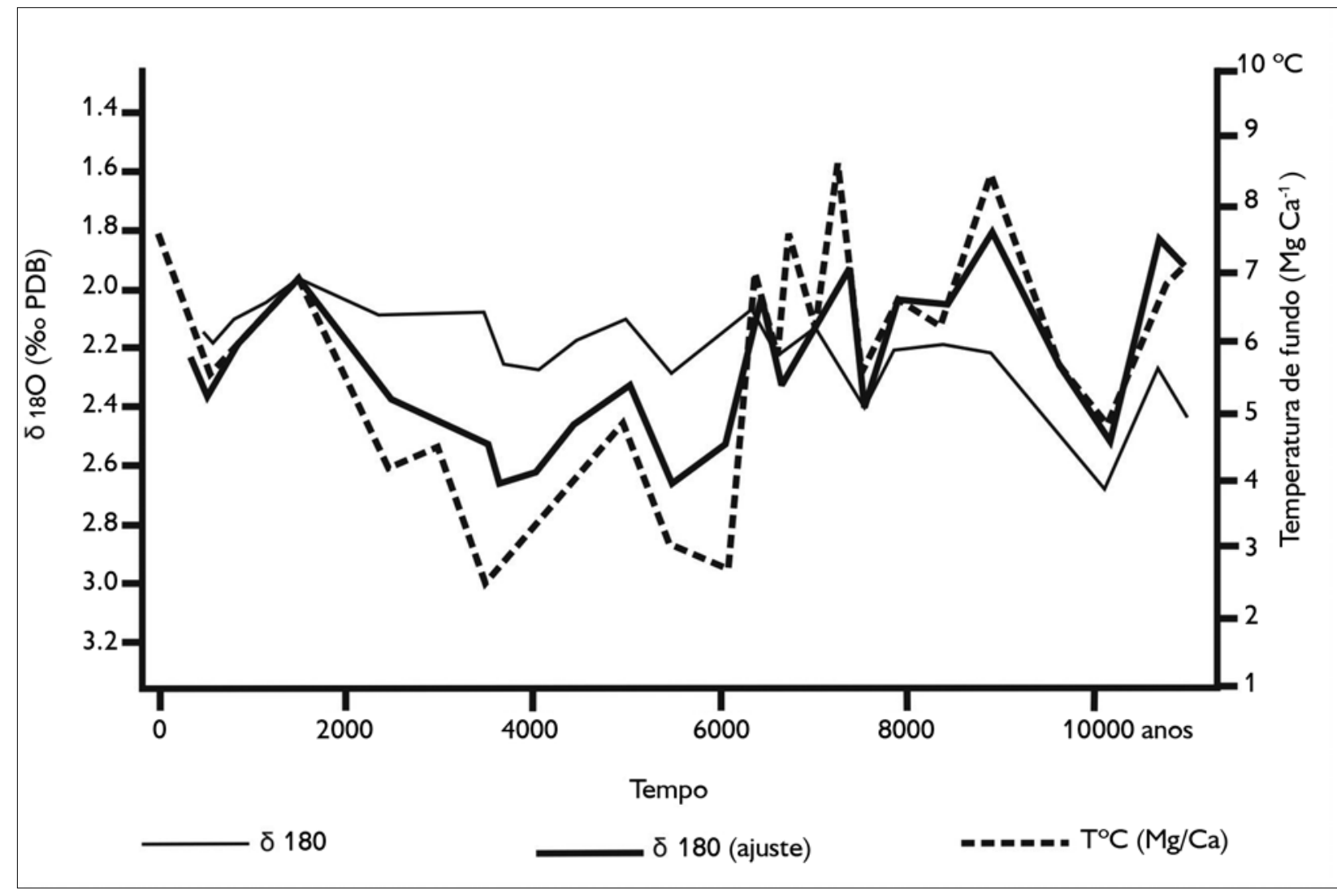

Figura 3. Curva de temperatura com base em $\mathrm{Mg} \mathrm{Ca}{ }^{-1}$ em ostracodes comparada com curva isotópica em Cibicidoides, no Atlântico Norte. Modificado de Dwyer et al. (2000).

hidrológicos, delimitando a região de abrangência de uma massa d'água. O grupo de espécies de foraminíferos, denominado 'Plexo Globorotalia menardii', é muito utilizado em estudos no Quaternário. Dados provenientes deste plexo aprimoraram o conhecimento sobre os eventos oceanográficos ocorridos na bacia de Campos, no sudeste do Brasil, durante o Pleistoceno (Vicalvi, 1997). Numa abordagem mais ampla, Aguiar et al. (2002) constataram a influência da mistura de águas tropicais e subtropicais, provenientes da Corrente das Malvinas e Corrente do Brasil, respectivamente, na fauna total de foraminíferos planctônicos no sul do Brasil.

Há também estudos desenvolvidos no Cretáceo e Paleogeno demonstrando o potencial paleoceanográfico destes fósseis. As variações morfológicas observadas em foraminíferos planctônicos permitiram a Koutsoukos (1996) dimensionar a influência da reorganização oceanográfica no Eocenozóico naqueles organismos. Num bom exemplo de integração de elementos planctônicos, Cunha \& Koutsoukos (1998) caracterizaram o panorama paleoceanográfico da bacia do Sergipe, no Aptiano, com base em sucessões de nanofósseis e foraminíferos.

Já a integração entre grupos planctônicos e bentônicos fornece, simultaneamente, informações sobre as massas d'água superficiais e de fundo.Variações de abundância do nanofóssil Florisphaera profunda, por exemplo, são indicadoras da profundidade da termoclina. Toledo et al. (2007a) e Toledo et al. (2007b) inferiram a influência das mudanças climáticas dos últimos 25 Ka na região da bacia de Santos. Empregando nanofósseis calcários e análises

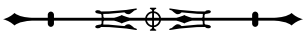




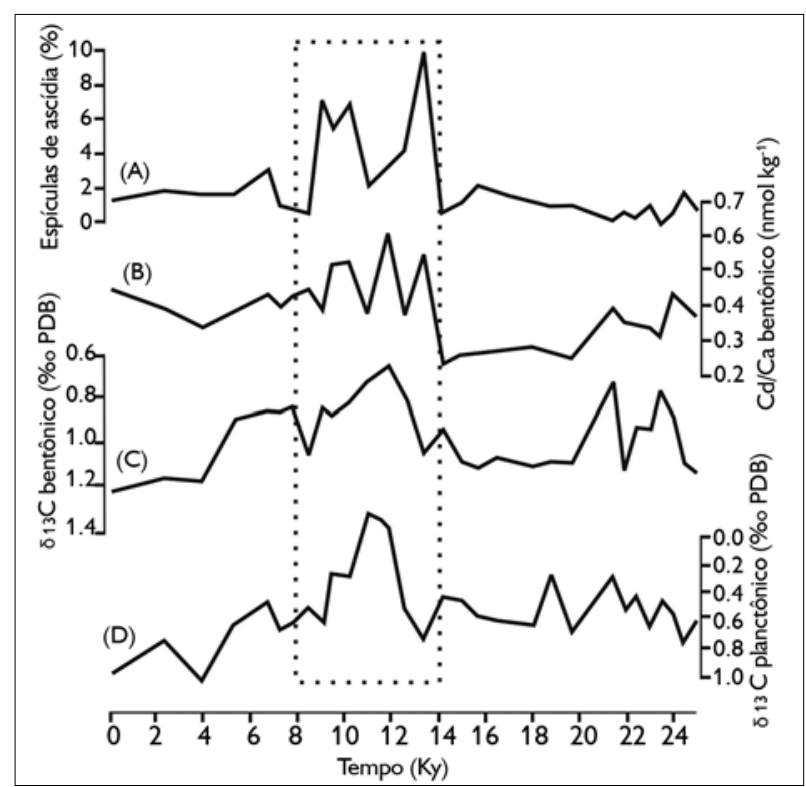

Figura 4. Variações de abundância de nanofósseis calcários (espículas de ascídias) e curvas isotópicas (carbono e oxigênio) e de elementos-traço $\left(\mathrm{Cd} \mathrm{Ca}^{-1}\right)$, em foraminíferos. Modificado de Toledo et al. (2007a).

isotópicas em foraminíferos bentônicos e planctônicos, registraram oscilações de produtividade e temperatura, pois a composição nanoflorística e a caracterização quantitativa de determinados gêneros de nanofósseis calcários são excelentes indicadores oceanográficos. Uma revisão sobre os estudos realizados e o estado da pesquisa com nanofósseis quaternários no Brasil pode ser encontrada em Antunes (2007).

Diferente dos microfósseis planctônicos, os ostracodes indicam apenas condições do fundo oceânico. Vários estudos publicados nas duas últimas décadas relacionam associações de espécies à estrutura hidrológica e circulação oceânica (Cronin et al., 1999 e Ayress et al., 2004, por exemplo). Steineck \& Thomas (1996) e Speijer \& Morsi (2002) discutem as mudanças oceanográficas decorrentes de aquecimento no período Paleoceno-Eoceno e sua influência nos ostracodes de Maud Rise (Mar de Weddell) e no norte da África, respectivamente. Dall'Antonia (2003), por sua vez, desenvolveu um estudo semelhante com faunas miocênicas do Mediterrâneo Central, onde observou a transição de uma fase termosférica para uma psicrosférica, com base na ocorrência do gênero criofilico Agrenocythere. Estudos similares foram também desenvolvidos com faunas cretáceas e, de forma análoga aos foraminíferos, Majoran et al. (1997) propuseram o cálculo de taxas de acumulação de valvas (OVAR - 'Ostracode Valve Accumulation Rates') como um indicador paleoceanográfico.

Além da interpretação da associação total, alguns gêneros são utilizados isoladamente como indicadores paleoambientais, em virtude das preferências ecológicas. O estudo de Peypouquet (1979) constitui uma referência clássica nesta linha, postulando que a morfologia do vestíbulo (estrutura formada entre as lamelas externa e interna da carapaça dos ostracodes) do gênero Krithe varia conforme a concentração de oxigênio. Esta hipótese foi testada por outros autores, alguns dos quais a refutaram, afirmando não encontrar relações consistentes entre a morfologia do vestíbulo e os níveis de oxigênio do meio. Mckenzie et al. (1989), Whatley \& Quanhong (1993) e Van Harten (1996), por exemplo, registraram, dentro de uma mesma associação, espécies de Krithe com diferentes morfologias de vestíbulo, não relacionáveis, segundo eles, aos níveis de oxigênio do meio.

Mais recentemente, ostracodes da família Cytherellidae passaram também a ser estudados como possíveis indicadores de níveis de oxigenação, e uma síntese sobre o tema pode ser encontrada em Whatley et al. (2003). Diversos estudos apontam um aumento de abundância de espécies do gênero Cytherella em eventos disaeróbicos, indicando a existência de possíveis vantagens adaptativas em ambientes depletados em oxigênio. Além dos eventos disaeróbicos, aumentos na abundância de Cytherella são também observados na Zona de Mínimo de Oxigênio (ZMO) dos oceanos atuais. Acredita-se que este padrão de abundância esteja relacionado com peculiaridades morfológicas da biologia reprodutiva de Cytherella (Corbari et al., 2005). Contudo, autores como Swanson et al. (2005) afirmam que, possivelmente, outros fatores determinam a abundância 
destes ostracodes, o que mostra que este tema merece ainda ser melhor estudado.

Recentemente, estudos com ostracodes sob enfoque paleoceanográficos vêm se difundindo em instituições brasileiras. Carreño et al. (1999) identificaram ciclos transgressivos-regressivos neocenozóicos na bacia de Pelotas, com base na caracterização e mudanças faunísticas. Mais recentemente, o comportamento das faunas de ostracodes batiais durante os eventos Neopleistocênicos, através de dados faunísticos e geoquímicos, vem sendo estudado por Bergue et al. (2006) e Bergue et al. (2007), na bacia de Santos, e Nicolaidis (2008), na bacia de Campos.

\section{CONSIDERAÇÕES FINAIS}

A revisão aqui apresentada fornece uma síntese de como os microfósseis calcários podem contribuir para a elucidação das mudanças climáticas ocorridas no passado. A vasta margem continental brasileira apresenta uma série de bacias com seções depositadas desde o Eocretáceo, sob os mais diversos contextos ecológicos, e que registram a história do Atlântico Sul Ocidental. Algumas delas, como Santos e Campos, são relativamente bem estudadas em termos paleoceanográficos, especialmente no intervalo Neocenozóico, em outras, porém, estas pesquisas são ainda inexistentes. Muito mais do que subsídios para a indústria, esta pesquisa pode aprimorar o conhecimento paleontológico dos microfósseis, além de contribuir para a compreensão dos processos sedimentares e de mudanças na formação e circulação nas massas d'água ao longo do tempo. Em suma, tem a possibilidade de proporcionar uma melhor compreensão da dinâmica do nosso planeta e de como a ação humana pode afetar os sistemas marinhos e, conseqüentemente, o clima da Terra.

\section{AGRADECIMENTOS}

Os autores agradecem a Felipe Antônio de Lima Toledo (IO-USP), Thomas Cronin (USGS) e Ilaria Mazzini (Ludwig Maximilians Universität) pelas Figuras 2, 3 e 4, gentilmente cedidas para ilustrar este trabalho. Agradecem ainda ao prof. Marco Antônio F. Hansen (UNISINOS), a Adriana Leonhardt (PPGeo-UFRGS) e aos revisores, pela leitura crítica e valiosas sugestões que contribuíram para o aperfeiçoamento do manuscrito.

\section{REFERÊNCIAS}

AGUiAR, E. S., I. I. LEIPNITZ \& E. L. C. LAVINA, 2002. Distribuição zoogeográfica dos foraminíferos palanctônicos do Quaternário da plataforma externa e talude superior $\left(30^{\circ} \mathrm{S}-34^{\circ} \mathrm{S}\right.$ e $49^{\circ} \mathrm{W}-52^{\circ} \mathrm{W}$ ) do estado do Rio Grande do Sul. Revista Brasileira de Paleontologia 4: 5-12.

ANTUNES, R. L., 2007. Nanofósseis calcários do Quaternário da margem continental Brasileira. Série Ciência Técnica-Petróleo 21: $1-75$.

AYRESS, M., P. DE DECKKER \& G. COLES, 2004. A taxonomic and distributional survey of marine benthonic Ostracoda off Kerguelen and Heard islands, South Indian Ocean. Journal of Micropalaeontology 23: 15-38.

BERGUE, C. T., J. C. COIMBRA \& T. M. CRONIN, 2007. Cytherellid species (Ostracoda) and their significance to the Late Quaternary events in the Santos Basin, Brazil. Senckenbergiana Maritima 37(1): 5-12.

BERGUE, C. T., K. B. COSTA, G. DWYER \& C. A. V. MOURA, 2006. Bathyal ostracode diversity in the Santos Basin, Brazilian southeast margin: response to Late Quaternary climate changes. Revista Brasileira de Paleontologia 9(2): 15-26.

CARREÑO, A. L., J. C. COIMBRA \& D. A. CARMO, 1999. Late Cenozoic sea level changes evidenced by ostracodes in the Pelotas Basin, southernmost Brazil. Marine Micropaleontology 37: 117-129.

CORBARI, L., N. MESMER-DUDONS, P. CARBONEL \& J. C. MASSABUAU, 2005. Cytherella as a tool to reconstruct deep-sea paleooxygen levels: the respiratory physiology of the platycopid ostracod Cytherella of. abyssorum. Marine Biology 147(6): 1377-1386.

CORRÈGE, T. \& P. DE DECKKER, 1997. Faunal and geochemical evidence for changes in intermediate water temperature and salinity in the western Coral Sea (NortheastAustralia) during the Late Quaternary. Palaeogeography, Palaeoclimatology, Palaeoecology 313: 183-205.

COSTA, K. B., F. A. L. TOLEDO, M. A. G. PIVEL \& F. CHEMALE JR., 2006. Evaluation of two genera of benthic foraminifera for down-core paleotemperature studies in the western south Atlantic. Brazilian Journal of Oceanography 54(1): 75-84.

CRONIN, T. M., R. THUNELL, G. DWYER, C. SAENGER, M. E. MANN, C. VANN \& R. R. SEAL, 2005. Multiproxy evidence of Holocene climate variability from estuarine sediments, eastern North America. Paleoceanography, 20, PA4006, doi: 10.1029/2005 PA 001145. 
CRONIN, T. M., D. M. DE MARTINO, G. DWYER \& I. RODRIGUEZ-LÁZARO, 1999. Deep-sea ostracode species diversity: response to late Quaternary climate change. Marine Micropaleontology 37: 231-249.

CUNHA, A. S. \& E. A. M. KOUTSOUKOS, 1998. Calcareous nannofossils and planktic foraminifers in the upper Aptian of the Sergipe Basin, northeastern Brazil: palaeoecological inferences. Palaeogeography, Palaeoclimatology, Palaeoecology 142: 175-184.

DALL'ANTONIA, B., 2003. Deep sea ostracods as indicators of paleoceanographic changes: a case history from the middle-late Miocene of southern Italy (Central Mediterranean). Terra Nova 15: 52-63.

DIDIÉ, C. \& H. A. BAUCH, 2002. Implications of Upper Quaternary stable isotope records of marine ostracodes and benthic foraminifers for palaeocological and palaeoceanographical investigations. In: J. A. HOLMES \& A. R. CHIVAS (Eds.): The Ostracoda: applications in Quaternary research: 279-301. American Geophysical Union (Geophysical Monograph, 131), Washington.

DWYER, G., T. M. CRONIN, P. A. BAKER \& J. RODRIGUEZLAZARO, 2000. Changes in North Atlantic deep-sea temperature during climatic fluctuations of the last 25,000 years based on ostracode $\mathrm{Mg} / \mathrm{Ca}$ ratios. Geochemistry, Geophysics, Geosystems (AGU eletronic journal: http//g-cubed.org/).

DWYER, G., T. M. CRONIN, P. A. BAKER, M. E. RAYMO, J. S. BUZAS \& T. CORRĖGE, 1995. North Atlantic deep water temperature change during Late Pliocene and Late Quaternary climatic changes. Science 270: 1347-1351.

EMILIANI, C., 1955. Pleistocene temperatures. Journal of Geology 63: 538-578.

KOUTSOUKOS, E. A. M., 1996. Phenotypic experiments into new pelagic niches in early Danian planktonik foraminifera: aftermath of the K/T boundary event. In: M. B. HART (Ed.): Biotic Recovery from Mass Extinction Events. Geological Society Special Publication 102: 319-335.

LANÇONE, R. B., W. DULEBA \& M. MAHIQUES, 2005. Dinâmica de fundo da enseada do Flamengo, Ubatuba, Brasil, inferida a partir da distribuição espacial, morfometria e tafonomia de foraminíferos. Revista Brasileira de Paleontologia 8(3): 181-192.

LEAR, C. H., H. ELDERFIELD \& P. A. WILSON, 2000. Cenozoic deep sea temperatures and global ice volumes from $\mathrm{Mg} / \mathrm{Ca}$ in benthic foraminiferal calcite. Science 287: 269-272.

LOVELOCK, J., 2006. Gaia: cura para um planeta doente: 1-192. Cultrix, São Paulo.

MAIORAN, S., J. G. V. WIDMARK \& M. KUCERA, 1997. Palaeocological preferences and geographical distribution of Late maastrichtian deep-sea ostracodes in the South Atlantic. Lethaia 30: 53-64.
MAZZINI, I., 2005. Taxonomy, biogeography and ecology of Quaternary benthic Ostracoda (Crustacea) from circumpolar deep water of the Emerald basin (Southern Ocean) and the $S$ Tasman Rise (Tasman Sea). Senckenbergiana Maritima 35(1): 1-119.

MCKENZIE, K. G., S. MAJORAN, V. EMAMI \& R. A. REYMENT, 1989. The Krithe problem - first test of Peypouquet's hypothesis, with a redescription of Krithe praetexta praetexta (Crustacea, Ostracoda). Palaeogeography, Palaeoclimatology, Palaeoecology 74: 343-354.

NICOLAIDIS, D. D., 2008. Ostracodes de águas profundas do Pleistoceno/Holoceno da bacia de Campos: isótopos estáveis de oxigênio vs. mudanças faunísticas: 1-66. Dissertação (Mestrado em Geociências) - Universidade Federal do Rio Grande do Sul, Porto Alegre.

PEYPOUQUET, J. P., 1979. Ostracodes et paléoenvironnements. Méthodologie et application aux domaines profonds du Cénozoïque. Bulletin du Bureau de Recherches Géologiques et Minieres 4: 3-79.

PISIAS, N. G., D. G. MARTINSON, T. C. MOORE, N. J. SHACKLETON, W. PRELL, J. HAYS \& J. BODEN, 1984. High resolution stratigraphic correlation of benthic oxygen isotopic records spanning the last 300.000 years. Marine Geology 56: 119-136.

SOUSA, S. H. M., R. F. PASSOS, M. FUKUMOTO, I. C. A. SILVEIRA, R. C. L. FIGUEIRA, E. A. M. KOUTSOUKOS, M. M. MAHIQUES \& C. E. REZENDE, 2006. Mid-lower bathyal benthic foraminifera of the Campos Basin, southeast Brazilian margin: biotopes and controlling ecological factors. Marine Micropaleontology 61: 40-57.

SPEIJER, R. \& A. M. MORSI, 2002. Ostracode turnover and sea level changes associated with the Paleocene-Eocene thermal maximum. Geology 30(1): 23-26.

STEINECK, P. L. \& E. THOMAS, 1996. The latest Paleocene crisis in the deep sea: Ostracode sucession at Maud Rise, Southern Ocean. Geology 24(7): 583-586.

SWANSON, K. M., T. JELLINEK \& H. MALZ, 2005. The platycopine condition: new observations on reproduction, respiration and feeding in living, deep-sea Platycopina (Crustacea, Ostracoda). Senckenbergiana Maritima 35(2): 157-187.

THOMAS, F. C., F. S MEDIOLI \& D. B. SCOTT, 1990. Holocene and Latest Wisconsian benthic foraminiferal assemblages and paleocirculation history, lower Scotian slope and rise. Journal of Foraminiferal Research 20(3): 212-245.

TOlEDO, F. A. L., M. CACHÃO, K. B. COSTA \& M. A. G. PIVEL, 2007a. Planktonic foraminifera, calcareous nannoplankton and ascidian variations during the last $25 \mathrm{Kyr}$ in the Southwestern Atlantic: A paleoproductive signature. Marine Micropaleontology 64: 67-79.

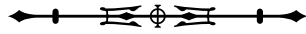


TOLEDO, F. A. L., K. B. COSTA \& M. A. G. PIVEL, 2007b. Salinity changes in the western tropical South Atlantic in the last $30 \mathrm{Ky}$. Global and Planetary Change 57: 383-395.

UREY, H. C., 1947. The thermodynamic properties of isotopic substances. Journal of Chemical Society 69: 562-581.

VAN HARTEN, D., 1996. The case against Krithe as a tool to estimate the depth and oxygenation of ancient oceans. In: A. MOGUILEVSKY \& R. C. WHATLEY (Eds.): Microfossils and Oceanic Environments: 297-304. Elsevier, Amsterdam.

VICALVI, M. A., 1997. Zoneamento bioestratigráfico e paleoclimático dos sedimentos do Quaternário superior do talude da bacia de Campos, RJ, Brasil. Boletim de Geociências da PETROBRAS 11(1-2): 132-165.
WHATLEY, R. C., R. PYNE \& I. P. WILKINSON, 2003. Ostracoda and paleo-oxygen levels, with particular reference to the Upper Cretaceous of East Anglia. Palaeogeography, Palaeoclimatology, Palaeoecology 194: 355-386.

WHATLEY, R. C. \& Z. QUANHONG, 1993. The Krithe problem: a case history of the distribution of Krithe and Parakrithe (Crustacea; Ostracoda) in the South China sea. Palaeogeography, Palaeoclimatology, Palaeoecology 103: 281-297.

Recebido: 27/07/2007

Aprovado: 22/09/2008 